\title{
REVIEW ARTICLE Involvement of the secreted protein Metrnl in human diseases
}

\author{
Zhu-wei Miao ${ }^{1}$, Wen-jun $\mathrm{Hu}^{1}$, Zhi-yong $\mathrm{Li}^{1}$ and Chao-yu Miao ${ }^{1}$
}

Metrnl, a secreted protein expressed in white adipose tissue, has been identified as a novel adipokine. It is also highly expressed in barrier tissues, including the skin, intestinal and respiratory tract epithelium in both mice and humans. Research shows that its expression is upregulated by inflammation, chronic high-fat diets, exercise, cold exposure, etc., and it plays important roles in promoting neurite extension, enhancing white fat browning, improving insulin sensitivity, modulating lipid metabolism and regulating inflammatory response, the latter implying Metrnl is a new cytokine. These studies suggest that Metrnl could be a promising biomarker and a potential therapeutic target for the related diseases. For proving this, clinical studies need to be performed to bridge the gap between bench and bedside. In this paper, we summarize the progress in recent clinical research on Metrnl. Most of these clinical studies are designed to confirm the relationship between circulating Metrnl and metabolic or cardiovascular disease (type 2 diabetes and coronary heart disease), or immune inflammation-related diseases, such as colitis, psoriasis and arthritis. Although blood Metrnl seems to fluctuate and are affected by many factors, such as drugs, physical exercise, and cold exposure, these clinical studies provide reliable clues that Metrnl is associated with coronary heart disease, inflammationrelated diseases, etc. Nevertheless, the roles of Metrnl in some diseases such as nervous system diseases remain unclear, and its putative involvement should be further clarified. These studies could promote the application of Metrnl in clinic as a new therapeutic target.

Keywords: secreted protein; adipokine; Metrnl; type 2 diabetes; obesity; coronary heart disease; atherosclerosis; colitis; psoriasis; arthritis

Acta Pharmacologica Sinica (2020) 41:1525-1530; https://doi.org/10.1038/s41401-020-00529-9

\section{INTRODUCTION}

Secreted proteins play a crucial role in both pathological and physiological processes and have a natural advantage for application in the diagnosis and treatment of clinical diseases [1]. Metrnl (Meteorin-like, Subfatin, Cometin) was previously discovered by us as an adipokine [2] and is abundant in subcutaneous white adipose tissue [2] and barrier tissues [3], such as the skin, intestinal, and respiratory tract epithelium [4].

Research has shown that Metrnl can promote neurite extension [5], enhance white fat browning [6], regulate insulin sensitivity [7], modify the inflammatory immune response [3], alter blood lipids [8], etc. Most of the research on the function of Metrnl has been reviewed by us before [9]. These results suggest that Metrnl could be a promising biomarker and a potential therapeutic target for related clinical diseases. However, the exact relationship between Metrnl and related clinical diseases needs to be confirmed, and whether Metrnl could be applied in the diagnosis and treatment of clinical diseases should be carefully verified.

Recently, many clinical studies related to Metrnl have been carried out. Since Metrnl is abundantly expressed in metabolismrelated organ and barrier tissues and shows regulatory functions in metabolism and inflammation in animal studies $[3,6,7,10]$, most clinical studies focus on the relationship between Metrnl and metabolism or inflammation-related diseases, such as type 2 diabetes [11-13], coronary heart disease [14, 15], colitis [16], arthritis [3, 17], etc. All these clinical studies are helpful for understanding the clinical effects of Metrnl and demonstrate its potential as a new therapeutic target. Here, in this paper, we will mainly introduce the progress of recent clinical research on Metrnl.

\section{METRNL IN TYPE 2 DIABETES MELLITUS}

Adipokines are synthesized and secreted by adipose tissue and participate in many physiological functions, such as energy metabolism, cardiovascular function, immune inflammation, and insulin sensitivity $[18,19]$. We have identified Metrnl as an adipokine since it is highly expressed in subcutaneous white fat and can be released by adipose tissue [2]. We have demonstrated that adipose Metrnl can promote adipose remodeling, adipocyte differentiation and lipid metabolism and regulate insulin sensitivity through the PPARY pathway in mice [7]. Meanwhile, Rao et al. showed that Metrnl can enhance energy expenditure and improve glucose tolerance by promoting white adipose tissue browning [6]. These animal studies have led to many researchers verifying the role of Metrnl in clinical metabolic diseases. Most of the studies focus on the relationship between type 2 diabetes and Metrnl serum levels. However, there are conflicting data in clinical studies regarding circulating Metrnl levels in type 2 diabetes mellitus.

\footnotetext{
${ }^{1}$ Department of Pharmacology, Second Military Medical University/Naval Medical University, Shanghai 200433, China

Correspondence: Chao-yu Miao (cymiao@smmu.edu.cn) or Zhi-yong Li (zhiyongli@smmu.edu.cn)

These authors contributed equally: Zhu-wei Miao, Wen-jun Hu
}

Received: 7 May 2020 Accepted: 5 September 2020

Published online: 30 September 2020 
Table 1. The clinical research on the relationship between blood Metrnl and type 2 diabetes.

\begin{tabular}{|c|c|c|c|}
\hline Authors/year & Sample size & Included criteria & Conclusion \\
\hline $\begin{array}{l}\text { Dadmanesh et al. } \\
\text { (2018) [14] }\end{array}$ & $\begin{array}{l}170 \text { subjects ( } 66 \text { patients with CAD, } 63 \\
\text { T2D patients and } 41 \text { controls) }\end{array}$ & $\begin{array}{l}\text { Patients with }>70 \% \text { stenosis in at least one } \\
\text { coronary artery were diagnosed as CAD. } \\
\text { Participants with history and evidence of } \\
\text { stroke, myocardial infarction, etc. or using } \\
\text { thiazolidinedione family drugs were } \\
\text { excluded }\end{array}$ & $\begin{array}{l}\text { Serum Metrnl levels were lower in CAD } \\
\text { and T2D patients compared to the } \\
\text { control group }\end{array}$ \\
\hline $\begin{array}{l}\text { Zheng et al. } \\
\text { (2018) [22] }\end{array}$ & $\begin{array}{l}20 \text { subjects ( } 11 \text { healthy controls, } 9 \\
\text { patients with newly diagnosed T2D) }\end{array}$ & No other major diseases and treatment & $\begin{array}{l}\text { Circulating Metrnl in people with newly } \\
\text { diagnosed T2D was lower }\end{array}$ \\
\hline $\begin{array}{l}\text { Chung et al. } \\
\text { (2018) [13] }\end{array}$ & $\begin{array}{l}800 \text { subjects ( } 400 \text { patients with T2D and } \\
400 \text { non-diabetes) }\end{array}$ & $\begin{array}{l}\text { Over } 40 \text { years of age without history of } \\
\text { cardiovascular disease, without stage } 2 \\
\text { hypertension, malignant disease, severe } \\
\text { renal or hepatic disease }\end{array}$ & $\begin{array}{l}\text { Blood Metrnl increased in patients } \\
\text { with T2D }\end{array}$ \\
\hline $\begin{array}{l}\text { Wang et al. (2019) } \\
\text { [11] }\end{array}$ & $\begin{array}{l}160 \text { subjects ( } 40 \text { subjects with normal } \\
\text { glucose tolerance, } 40 \text { subjects with } \\
\text { impaired fasting glucose, } 40 \text { subjects with } \\
\text { impaired glucose tolerance, and } 40 \\
\text { patients newly diagnosed T2D) }\end{array}$ & $\begin{array}{l}\text { Patients with previously diagnosed T2D, } \\
\text { other types of diabetes, other major } \\
\text { diseases, and medication history including } \\
\text { the use of antidiabetics, statins, diuretics, } \\
\text { corticosteroids, estrogen and progestin } \\
\text { were excluded }\end{array}$ & $\begin{array}{l}\text { Blood Metrnl was highest in patients } \\
\text { with T2D and significantly increased in } \\
\text { patients with prediabetes compared } \\
\text { with individuals with normal glucose } \\
\text { tolerance }\end{array}$ \\
\hline $\begin{array}{l}\text { El-Ashmawy et al. } \\
\text { (2019) [23] }\end{array}$ & $\begin{array}{l}260 \text { subjects ( } 89 \text { subjects with normal } \\
\text { glucose tolerance, } 77 \text { subjects with } \\
\text { glucose tolerance impairment and } 94 \\
\text { with T2D) }\end{array}$ & $\begin{array}{l}\mathrm{BMI}<35 \mathrm{~kg} / \mathrm{m}^{2} \text {; age between } 20 \text { and } 75 \\
\text { years; no other CVD; no history of } \\
\text { malignancy or recent infection; no history } \\
\text { of taking antidiabetic medications, } \\
\text { concomitant medications such as systemic } \\
\text { steroids, cholestyramine, statins, diuretics, } \beta \text { - } \\
\text { blockers or oral anticoagulants }\end{array}$ & $\begin{array}{l}\text { Serum Metrnl levels decreased in } \\
\text { patients with T2D versus subjects with } \\
\text { normal glucose tolerance }\end{array}$ \\
\hline
\end{tabular}

T2D type 2 diabetes, $C A D$ coronary artery disease, $C V D$ cardiovascular diseases, $B M I$ body mass index.

As shown in Table 1, some studies show that blood Metrnl levels are increased in type 2 diabetes when compared with nondiabetes, including the studies of Chung et al. [13], AlKhairi et al. [20], Wang et al. [11], etc., whereas other studies have drawn the opposite conclusion that blood Metrnl is reduced in type 2 diabetes, including the studies of Lee et al. [21], Dadmanesh et al. [14] and our laboratory [22]. Consistent with the reduced Metrnl level in type 2 diabetes, El-Ashmawy et al.'s study also showed that blood Metrnl is negatively correlated with HOMA-IR and HbA1c [23].

The difference in these results may be caused by many influencing factors in clinical investigations, such as various treatments the patients received, disease severity, etc. One of the major factors is the drugs taken by patients. The results from Lee et al.'s research show that 12 weeks of treatment with metformin does not change blood Metrnl [21], while Liu et al. show that antihyperglycemic therapy slightly increases serum Metrnl concentrations without statistical significance [15]. Based on our unpublished data, 3 months of treatment with metformin or glucagon-like peptide 1 (GLP-1) does not change circulating Metrnl, but pioglitazone treatment can increase circulating Metrnl levels in mice fed a high-fat diet. In addition to antidiabetic drugs, lipid-lowering drugs may also alter blood Metrnl levels. In Chung et al.'s study, the total cholesterol, triglyceride, and low-density lipoprotein (LDL)-cholesterol levels of patients with type 2 diabetes were reduced [13], indicating that these patients may use lipid-lowering drugs, which could increase Metrnl secretion. This guess is also reflected in the data from AlKhairi et al.'s research [20] in which the total cholesterol of the patients is also reduced and blood Metrnl is increased.

As we mentioned above, antidiabetes treatments, including thiazolidinedione family drugs and physical exercise, could regulate blood Metrnl levels in humans. Clinical investigation of newly diagnosed patients with type 2 diabetes can avoid these interfering factors. Thus, the studies of Lee et al. [21], Zheng et al. [22], and Wang et al. [11] used newly diagnosed patients. The first two studies show that Metrnl is lower in newly diagnosed patients with type 2 diabetes patients, while the last one shows higher Metrnl in newly diagnosed patients with type 2 diabetes. These inconsistent results suggest that blood Metrnl fluctuates and is affected by many other factors, apart from antidiabetes medications. For example, cold exposure could increase Metrnl expression in adipose tissue in mice. Physical exercise (high-intensity interval exercise) could also obviously increase the muscle 
expression of Metrnl in humans, which may last for several days $[24,25]$. Thus, to avoid interfering factors, well-designed and controlled clinical studies with much larger sample sizes should be carried out before a credible conclusion can be drawn.

Circulating Metrnl may also be affected by disease severity. Diabetes is considered a chronic low-grade inflammatory disease, and inflammation could raise the blood level of Metrnl. Although Metrnl has indeed been verified to improve insulin sensitivity in animal experiments $[6,7]$, to the best of our knowledge, the relationship between blood Metrnl and type 2 diabetes has not been clearly clarified until now in animal models. It is also possible that severe type 2 diabetes with systemic inflammation could trigger the upregulation of Metrnl, leading to an increase in blood Metrnl in patients with severe diabetes. Therefore, to study the relationship between blood Metrnl and type 2 diabetes, a welldesigned cohort study is needed. In addition to excluding possible influencing factors, the severity of diabetes must be scored, such as rating the fluctuation degree of blood glucose, complications, etc.

\section{METRNL IN OBESITY}

Different results have also been obtained in research on the relationship between obesity and blood Metrnl. Previously, we found that Metrnl expression in adipose tissue and blood Metrnl levels were increased in obese mice. Consistently, Loffler et al. reported that Metrnl expression in adipose tissue is higher in obese children than in lean children [26], and AlKhairi et al. showed that blood Metrnl is increased in obese subjects [20]. However, we failed to find any relationship between serum Metrnl and BMI (body mass index) in subjects undergoing a physical examination [7], in line with the results from the study of Wang et al. [27]. Recently, several studies have shown a negative correlation between circulating Metrnl and BMI or visceral fat area $[14,28-30]$. This discrepancy may also result from species differences or confounding factors in clinical studies, such as age, sex, drugs, and physical activity.

\section{METRNL IN CORONARY HEART DISEASE AND ATHEROSCLEROSIS}

Coronary heart disease is one of the major causes of death worldwide and mainly arises from atherosclerosis [31]. The major risk factors for atherosclerosis include dyslipidemia, inflammation and insulin resistance [31, 32]. Previous research shows that Metrnl plays a role in regulating insulin resistance and inflammatory responses $[3,6,7]$. Our recent work shows that global knockout of Metrnl can not only increase blood triglycerides but also decrease high-density lipoprotein (HDL)-cholesterol in mice. Combined with the results from a tissue-specific knockout animal model, we conclude that adipose Metrnl contributes to its regulatory function on blood triglycerides and that liver Metrnl contributes to its regulatory effects on cholesterol metabolism [8]. Thus, it is speculated that a relationship between blood Metrnl and coronary heart disease could exist in the clinic.

Liu et al. showed that a decreased blood Metrnl concentration is significantly related to coronary heart disease in patients with diabetes or nondiabetics [15]. According to their regression analysis, the optimal cutoff value of Metrnl for predicting coronary heart disease is $123.5 \mathrm{pg} / \mathrm{mL}$, with a low sensitivity (44.04\%) and a high specificity (95.51\%). Meanwhile, they also carried out research on the relationship between Metrnl levels and the severity of coronary heart disease. The severity of coronary heart disease is assessed with the Gensini score system based on the degree of luminal narrowing and its geographic importance. The results show that serum Metrnl is significantly negatively correlated with the Gensini score and the number of stenosed vessels, indicating that lower Metrnl levels are associated with the severity of coronary heart disease [15]. A similar phenomenon was also observed in the study of Dadmanesh et al., which showed that blood Metrnl was significantly lower in patients with coronary heart disease [14]. These results suggest that low blood Metrnl is related to coronary heart disease and that Metrnl may have a protective role in coronary heart disease. There are some clues to explain the mechanism; for example, lower blood Metrnl is associated with LDL cholesterol, carotid intima media thickness, brachial-ankle pulse wave velocity, intercellular adhesion molecule 1 (ICAM-1), vascular cell adhesion molecule 1 (VCAM-1) and E selectin [23]. However, the comprehensive explanation of why lower blood Metrnl is associated with coronary heart disease and the exact function of Metrnl in coronary heart disease need to be explored further.

\section{METRNL IN COLITIS}

Metrnl is considered a cytokine and is also designated IL-39 or IL$41[3,17]$. It has been reported that Metrnl is closely related to inflammation-related diseases, including colitis and psoriasis [3]. Rao et al. first reported that Metrnl plays an anti-inflammatory effect via eosinophil-released IL-4 [6]. Metrnl expression and circulating concentrations are increased in inflammatory tissue or diseases [10]. Ushach et al. verified that Metrnl is highly expressed in IL-4/macrophage colony-stimulating factor (M-CSF)-induced M2 macrophages [3]. The interaction between Metrnl and other cytokines has been verified in cultured bone marrow macrophages. On the one hand, inflammatory factors can regulate Metrnl expression. For example, TNFa, IL-17a, IL-12, IL-4, and IL-1 $\beta$ can increase Metrnl expression, while IFN $\gamma$ and TGF $\beta$ can inhibit its expression. On the other hand, Metrnl can also regulate the expression of other inflammatory factors in macrophages, such as IL-10, IL-6, CCL2, CXCL1, etc.; inhibit the expression of MHC class II in peritoneal macrophages; and affect immunoglobulin class switching $[3,10]$. We and Ushach et al. [10] found that the Metrnl concentration in blood is dramatically increased in a sepsis animal model induced by endotoxin, and Metrnl deficiency increases susceptibility to endotoxin-induced mortality in mice. In a clinical study, serum Metrnl levels were weakly negatively correlated with high sensitivity $C$ reactive protein and IL-1 $\beta$ [15], IL- 6 and TNFa $[14,23]$ but weakly positively correlated with the antiinflammatory cytokine IL-11 in patients with coronary artery disease [15].

Previously, we demonstrated that Metrnl is highly expressed in intestinal epithelial cells both in humans and mice, but intestinal Metrnl does not contribute to circulating Metrnl; instead, it is mainly released into the intestinal lumen [4]. Furthermore, using a Metrnl intestinal-specific knockout animal model, we found that Metrnl deficiency deteriorates 3\% dextran sodium sulfate-induced ulcerative colitis by altering the expression of local inflammatory factors [33]. However, whether Metrnl from circulation or other tissue contributes to intestinal local inflammation has not been verified.

Recent works have shown that Metrnl derived from mesenteric adipose tissue could also be associated with colitis [16]. Zuo et al. found that Metrnl is highly expressed in mesenteric adipose tissue in mice and humans with colitis [16]. Treatment of IL-10 knockout mice with Metrnl protein not only significantly reduces mesenteric adipose weight/body weight, decreases the infiltration of F4/80positive macrophages, and enlarges the size of adipocytes but also inhibits inflammation, reduces IL- 6 and TNFa expression and prevents macrophage alteration from $M 2$ to $M 1$. They also found that the phosphorylation of the transcription factor STAT5 is upregulated, suggesting that the STAT5/PPARY pathway is involved in the Metrnl signaling pathway. These animal experiments have clearly shown that Metrnl is closely related to colitis and has a beneficial role in the treatment of colitis. Hence, Metrnl is quite promising as a therapeutic target for colitis in light of the 
current research, but the relationship between Metrnl and human colitis needs to be verified in further studies.

\section{METRNL IN PSORIASIS AND ARTHRITIS}

Metrnl is highly expressed in skin and mucous membranes and plays an important role in both innate and adaptive immunity [3]. Mice deficient in Metrnl are prone to inflammatory damage, such as inflammatory lesions in the uterus [10]. Therefore, it makes sense that Metrnl, which is highly expressed in skin lesions, is associated with inflammation. Consistently, Ushach et al. verified that Metrnl is highly expressed in inflammatory human skin diseases, such as psoriasis, prurigo nodularis, actinic keratosis, and atopic dermatitis, but not in basal or squamous cell carcinoma [3].

Metrnl is expressed in hypertrophic chondrocytes and osteoblasts lining trabecular bone surfaces and can inhibit osteoblast differentiation [34] and is upregulated in synovial membranes of human rheumatoid arthritis [3]. Recently, different kinds of arthritis, including rheumatoid arthritis, psoriatic arthritis, and osteoarthritis, were compared in Bridgewood et al.'s study [17]. The results show that Metrnl is upregulated in both the synovium and synovial fluid of patients with psoriatic arthritis and patients with rheumatoid arthritis. Given that the enthesis, the attachment site of the tendon and ligaments to bone, is the initial site of inflammation in patients with psoriatic arthritis [35], they focus on the inflammatory response in enthesis and show that Metrnl is abundantly expressed in stromal cells and is significantly increased under the synergistic induction of IL-17A/F and tumor necrosis factor [17]. These results indicate the important role of Metrnl in inflammatory skin and arthritis synovium. However, the mechanism of action needs further research.

\section{METRNL REGULATES THE INTRACELLULAR SIGNALING PATHWAY}

Several studies have explored the intracellular signaling pathway that mediates the functions of Metrnl, but these changes seem to vary due to the cell types (Fig. 1). In the nervous system, Meteorin, a Metrnl homolog, induces phosphorylation of ERK $1 / 2$ in satellite glia and activation of the JAK-STAT3 pathway to promote GFAPpositive glial differentiation [36, 37]. Consistent with these findings, inhibition of JAK-STAT3 and MEK-ERK with JAKil or U0126 also reduces Metrnl-induced neurite outgrowth [5], suggesting that Metrnl and Meteorin could share the same signaling pathway in cells of the nervous system. In naïve/M0-like bone marrow-derived macrophages, Metrnl recombinant protein also increases STAT3 phosphorylation, and a STAT3 inhibitor abrogates Metrnl-induced expression of anti-inflammatory markers [38]. However, in adipocytes, we failed to observe Metrnlinduced phosphorylation of STAT3; instead, we found that Metrnl improves systemic insulin resistance via the upregulation of PPARY [7].

In muscle cells, Metrnl could induce AMPK phosphorylation by increasing intracellular calcium to improve glucose metabolism. Metrnl upregulates the expression of GLUT4 via AMPK-mediated phosphorylation of HDAC5, a transcription factor of GLUT4, and increases GLUT4 translocation to the plasma membrane via AMPKmediated TBC1D1 phosphorylation. In addition, Metrnl also increases glucose uptake via the p38 MAPK pathway in an AMPK-mediated manner [39], indicating that AMPK plays a key role in the Metrnl-regulated intracellular signaling pathway in muscle cells. Apart from AMPK, it has also been reported that the upregulation of PPAR $\delta$ expression mediates the beneficial effects of Metrnl on insulin resistance and anti-inflammation [40], processes in which STAT3 could also be involved [38]. Although several signaling pathways are regulated by Metrnl, Metrnl is still an orphan ligand, and the receptor or proteins that directly interact with Metrnl remain unclear.

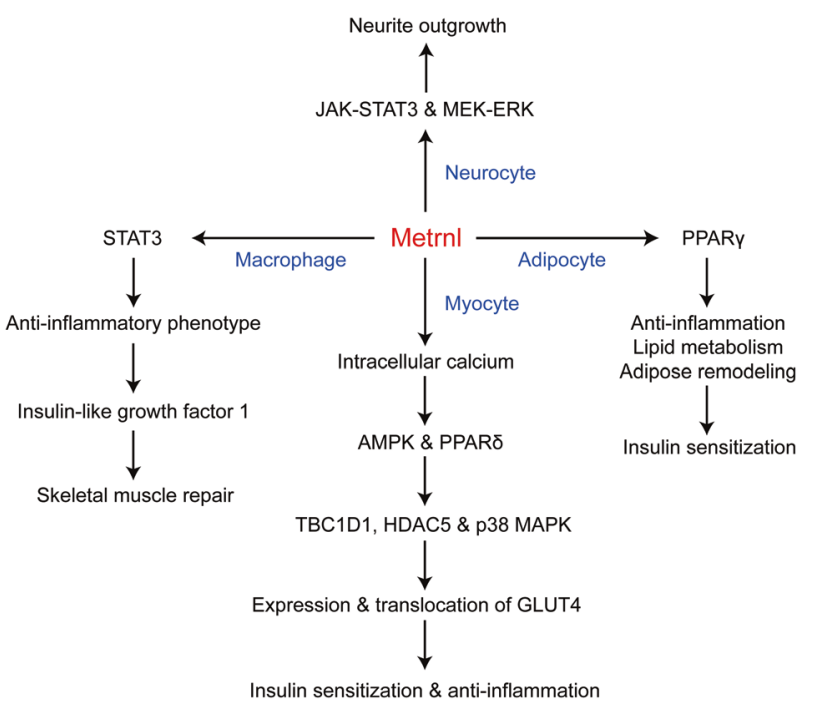

Fig. 1 Metrnl regulates the intracellular signaling pathway. In neurocytes, Metrnl protein promotes neurite outgrowth via the JAKSTAT3 and MEK-ERK signaling pathways. In adipocytes, overexpression of Metrnl enhances lipid metabolism, relieves high-fat dietinduced inflammation, and improves adipose remodeling through upregulation of PPAR $\gamma$, subsequently improving insulin resistance. In myocytes, Metrnl protein upregulates PPARס and promotes the phosphorylation of AMPK by increasing intracellular calcium and further promotes the phosphorylation of TBC1D1, HDAC5, and p38 MAPK in an AMPK-mediated manner, subsequently promoting expression and translocation of GLUT4, improving insulin sensitization and reducing inflammation. In naïve/MO-like bone marrowderived macrophages, Metrnl protein increases STAT3 phosphorylation, promotes macrophage differentiation to an anti-inflammatory phenotype, and releases insulin-like growth factor 1 , subsequently increasing primary muscle satellite cell proliferation and skeletal muscle repair.

\section{CONCLUSION}

Metrnl has shown emerging effects in some clinical diseases. In metabolic diseases, including type 2 diabetes mellitus, obesity, and dyslipidemia, changes in blood Metrnl seem to be related to the development of these diseases, but the exact relationship needs to be further confirmed with more detailed, well-designed and controlled clinical studies, especially cohort studies. In coronary heart disease, lower levels of blood Metrnl are associated with more severe coronary heart disease, and Metrnl itself may play a protective role in coronary heart disease. In colitis, psoriasis, and some arthritis, Metrnl expression is increased in these inflammatory diseases, and animal studies suggest that Metrnl treatment could relieve the symptoms of these diseases through local and systemic anti-inflammatory effects (Fig. 2).

Although clinical evidence shows that Metrnl is correlated with some metabolic and inflammatory diseases, the functions of Metrnl in humans need to be further verified, and the mechanisms are largely unknown. Clinical investigations between Metrnl and other human diseases in which Metrnl could be involved are still insufficient. For example, Metrnl is related to neurite extension and could be associated with the development of the central nervous system [5]. Ring chromosome 17 syndrome in which the Metrnl gene may be deleted is also associated with intellectual disability [41]. However, the exact function of Metrnl in the central nervous system in humans remains unknown. Apart from exploring Metrnl function in other diseases, a myriad of work still needs to be done to study the structure-function relationship of Metrnl and its regulatory mechanism in related diseases to develop it as a new therapeutic target. 


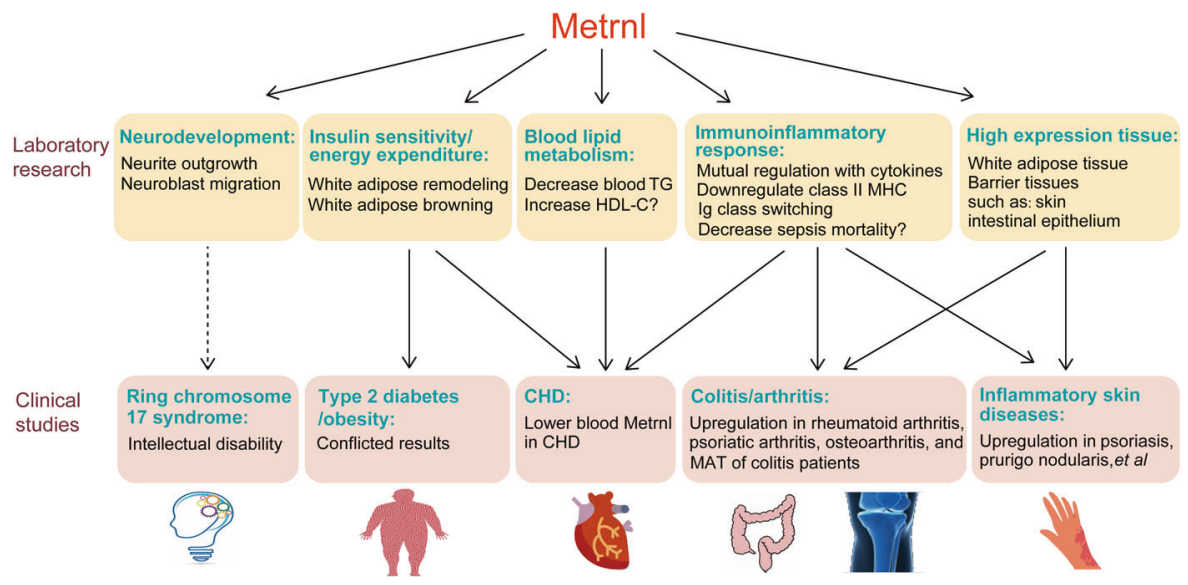

Fig. 2 The functions of Metrnl found by laboratory research and their corresponding clinical diseases. Laboratory research shows that Metrnl is highly expressed in white adipose tissue and barrier tissues and plays roles in neurodevelopment, insulin sensitivity, energy expenditure, blood lipid metabolism, and the immunoinflammatory response. Consistently, clinical studies indicate that Metrnl is associated with coronary heart disease (CHD), colitis, arthritis, and inflammatory skin diseases. Additional research should be done to clarify the relationship between Metrnl and ring chromosome 17 syndrome, type 2 diabetes, or obesity. TG triglyceride, HDL-C high-density lipoproteincholesterol, MAT mesenteric adipose tissue.

\section{ACKNOWLEDGEMENTS}

This work was supported by grants from the National Natural Science Foundation of China Major Project (No. 81730098), the Medical Innovation Project (No. 16QNP087), and the Shanghai Science and Technology Commission Project (No. 16JC1405100 and No. 201409004600).

\section{ADDITIONAL INFORMATION}

Competing interests: The authors declare no competing interests.

\section{REFERENCES}

1. Fasshauer M, Bluher M. Adipokines in health and disease. Trends Pharmacol Sci. 2015;36:461-70.

2. Li ZY, Zheng SL, Wang P, Xu TY, Guan YF, Zhang YJ, et al. Subfatin is a novel adipokine and unlike meteorin in adipose and brain expression. CNS Neurosci Ther. 2014;20:344-54.

3. Ushach I, Burkhardt AM, Martinez C, Hevezi PA, Gerber PA, Buhren BA, et al. METEORIN-LIKE is a cytokine associated with barrier tissues and alternatively activated macrophages. Clin Immunol. 2015;156:119-27.

4. Li ZY, Fan MB, Zhang SL, Qu Y, Zheng SL, Song J, et al. Intestinal metrnl released into the gut lumen acts as a local regulator for gut antimicrobial peptides. Acta Pharmacol Sin. 2016;37:1458-66.

5. Jorgensen JR, Fransson A, Fjord-Larsen L, Thompson LH, Houchins JP, Andrade N, et al. Cometin is a novel neurotrophic factor that promotes neurite outgrowth and neuroblast migration in vitro and supports survival of spiral ganglion neurons in vivo. Exp Neurol. 2012;233:172-81.

6. Rao RR, Long JZ, White JP, Svensson KJ, Lou J, Lokurkar I, et al. Meteorin-like is a hormone that regulates immune-adipose interactions to increase beige fat thermogenesis. Cell. 2014;157:1279-91.

7. Li ZY, Song J, Zheng SL, Fan MB, Guan YF, Qu Y, et al. Adipocyte metrnl antagonizes insulin resistance through PPARgamma signaling. Diabetes. 2015;64:4011-22.

8. Qi Q, Hu WJ, Zheng SL, Zhang SL, Le YY, Li ZY, et al. Metrnl deficiency decreases blood HDL cholesterol and increases blood triglyceride. Acta Pharmacol Sin. 2020. https://doi.org/10.1038/s41401-020-0368-8.

9. Zheng SL, Li ZY, Song J, Liu JM, Miao CY. Metrnl: a secreted protein with new emerging functions. Acta Pharmacol Sin. 2016;37:571-9.

10. Ushach I, Arrevillaga-Boni G, Heller GN, Pone E, Hernandez-Ruiz M, CatalanDibene J, et al. Meteorin-like/Meteorin-beta Is a novel immunoregulatory cytokine associated with inflammation. J Immunol. 2018;201:3669-76.

11. Wang K, Li F, Wang C, Deng Y, Cao Z, Cui Y, et al. Serum levels of Meteorin-Like (Metrnl) are increased in patients with newly diagnosed type 2 diabetes mellitus and are associated with insulin resistance. Med Sci Monit. 2019;25:2337-43.

12. Lappas M. Maternal obesity and gestational diabetes decrease metrnl concentrations in cord plasma. J Matern Fetal Neonatal Med. 2019:1-5. https://doi. org/10.1080/14767058.2019.1676713.
13. Chung HS, Hwang SY, Choi JH, Lee HJ, Kim NH, Yoo HJ, et al. Implications of circulating Meteorin-like (Metrnl) level in human subjects with type 2 diabetes. Diabetes Res Clin Pr. 2018;136:100-7.

14. Dadmanesh M, Aghajani $H$, Fadaei R, Ghorban K. Lower serum levels of Meteorinlike/Subfatin in patients with coronary artery disease and type 2 diabetes mellitus are negatively associated with insulin resistance and inflammatory cytokines. PLoS One. 2018;13:e0204180.

15. Liu ZX, Ji HH, Yao MP, Wang L, Wang Y, Zhou P, et al. Serum Metrnl is associated with the presence and severity of coronary artery disease. J Cell Mol Med. 2019;23:271-80.

16. Zuo L, Ge S, Ge Y, Li J, Zhu B, Zhang Z, et al. The Adipokine Metrnl ameliorates chronic colitis in II-10-/- mice by attenuating mesenteric adipose tissue lesions during spontaneous colitis. J Crohns Colitis. 2019;13:931-41.

17. Bridgewood C, Russell T, Weedon H, Baboolal T, Watad A, Sharif K, et al. The nove cytokine Metrnl/IL-41 is elevated in psoriatic arthritis synovium and inducible from both entheseal and synovial fibroblasts. Clin Immunol. 2019;208:108253.

18. Miao CY. Introduction: Adipokines and cardiovascular disease. Clin Exp Pharmacol Physiol. 2011;38:860-3.

19. Zhu X, Zhang HW, Chen HN, Deng XJ, Tu YX, Jackson AO, et al. Perivascular adipose tissue dysfunction aggravates adventitial remodeling in obese mini pigs via NLRP3 inflammasome/IL-1 signaling pathway. Acta Pharmacol Sin. 2019;40:46-54.

20. AlKhairi I, Cherian $P$, Abu-Farha $M$, Madhoun AA, Nizam $R$, Melhem $M$, et al. Increased expression of Meteorin-Like hormone in type 2 diabetes and obesity and its association with irisin. Cells. 2019;8:1283.

21. Lee JH, Kang YE, Kim JM, Choung S, Joung KH, Kim HJ, et al. Serum Meteorin-like protein levels decreased in patients newly diagnosed with type 2 diabetes. Diabetes Res Clin Pract. 2018;135:7-10.

22. Zheng SL, Li ZY, Zhang Z, Wang DS, Xu J, Miao CY. Evaluation of two commercial enzyme-linked immunosorbent assay kits for the detection of human circulating Metrnl. Chem Pharm Bull (Tokyo). 2018;66:391-8.

23. El-Ashmawy HM, Selim FO, Hosny TAM, Almassry HN. Association of low serum Meteorin like (Metrnl) concentrations with worsening of glucose tolerance, impaired endothelial function and atherosclerosis. Diabetes Res Clin Pract. 2019;150:57-63.

24. Eaton M, Granata C, Barry J, Safdar A, Bishop D, Little JP. Impact of a single bout of high-intensity interval exercise and short-term interval training on interleukin6 , FNDC5, and METRNL mRNA expression in human skeletal muscle. J Sport Health Sci. 2018;7:191-6.

25. Saghebjoo M, Einaloo A, Mogharnasi M, Ahmadabadi F. The response of meteorin-like hormone and interleukin-4 in overweight women during exercise in temperate, warm and cold water. Horm Mol Biol Clin Investig. 2018;36:/j/ hmbci.2018.36.issue-3/hmbci-2018-0027/hmbci-2018-0027.xml.

26. Loffler D, Landgraf $K$, Rockstroh D, Schwartze JT, Dunzendorfer $H$, Kiess W, et al. METRNL decreases during adipogenesis and inhibits adipocyte differentiation leading to adipocyte hypertrophy in humans. Int J Obes (Lond). 2017;41:112-9.

27. Wang C, Pan Y, Song J, Sun Y, Li H, Chen L, et al. Serum Metrnl level is correlated with insulin resistance, but not with beta-cell function in type 2 diabetics. Med Sci Monit. 2019;25:8968-74 
28. Pellitero S, Piquer-Garcia I, Ferrer-Curriu G, Puig R, Martinez E, Moreno P, et al. Opposite changes in meteorin-like and oncostatin $\mathrm{m}$ levels are associated with metabolic improvements after bariatric surgery. Int J Obes (Lond). 2018;42: 919-22.

29. Du Y, Ye X, Lu A, Zhao D, Liu J, Cheng J, et al. Inverse relationship between serum Metrnl levels and visceral fat obesity (VFO) in patients with type 2 diabetes. Diabetes Res Clin Pr. 2020;161:108068.

30. Fouani FZ, Fadaei R, Moradi N, Zandieh Z, Ansaripour S, Yekaninejad MS, et al. Circulating levels of Meteorin-like protein in polycystic ovary syndrome: a casecontrol study. PLoS One. 2020;15:e0231943.

31. Knapp M, Tu X, Wu R. Vascular endothelial dysfunction, a major mediator in diabetic cardiomyopathy. Acta Pharmacol Sin. 2019;40:1-8.

32. Li ZY, Wang P, Miao CY. Adipokines in inflammation, insulin resistance and cardiovascular disease. Clin Exp Pharmacol Physiol. 2011;38:888-96.

33. Zhang SL, Li ZY, Wang DS, Xu TY, Fan MB, Cheng MH, et al. Aggravated ulcerative colitis caused by intestinal Metrnl deficiency is associated with reduced autophagy in epithelial cells. Acta Pharmacol Sin. 2020;41:763-70.

34. Gong W, Liu Y, Wu Z, Wang S, Qiu G, Lin S. Meteorin-like shows unique expression pattern in bone and its overexpression inhibits osteoblast differentiation. PLoS One. 2016;11:e0164446.
35. Watad A, Cuthbert RJ, Amital H, McGonagle D. Enthesitis: much more than focal insertion point inflammation. Curr Rheumatol Rep. 2018;20:41.

36. Nishino J, Yamashita K, Hashiguchi H, Fujii H, Shimazaki T, Hamada H. Meteorin: a secreted protein that regulates glial cell differentiation and promotes axonal extension. EMBO J. 2004;23:1998-2008.

37. Lee HS, Han J, Lee SH, Park JA, Kim KW. Meteorin promotes the formation of GFAP-positive glia via activation of the Jak-STAT3 pathway. J Cell Sci. 2010;123:1959-68.

38. Baht GS, Bareja A, Lee DE, Rao RR, Huang R, Huebner JL, et al. Meteorin-like facilitates skeletal muscle repair through a Stat3/IGF-1 mechanism. Nat Metab. 2020;2:278-89.

39. Lee JO, Byun WS, Kang MJ, Han JA, Moon J, Shin MJ, et al. The myokine meteorinlike (metrnl) improves glucose tolerance in both skeletal muscle cells and mice by targeting AMPKalpha2. FEBS J. 2020;287:2087-104.

40. Jung TW, Lee SH, Kim HC, Bang JS, Abd El-Aty AM, Hacimuftuoglu A, et al. METRNL attenuates lipid-induced inflammation and insulin resistance via AMPK or PPARdeltadependent pathways in skeletal muscle of mice. Exp Mol Med. 2018;50:122.

41. Surace C, Piazzolla S, Sirleto P, Digilio MC, Roberti MC, Lombardo A, et al. Mild ring 17 syndrome shares common phenotypic features irrespective of the chromosomal breakpoints location. Clin Genet. 2009;76:256-62. 